\title{
SELF-ENERGY OF ELECTRONS IN NON-PERTURBATIVE QED
}

\author{
Elliott H. Lieb and Michael Loss
}

\begin{abstract}
Various models of charged particles interacting with a quantized, ultraviolet cutoff radiation field (but not with each other) are investigated. Upper and lower bounds are found for the self- or ground state-energies without mass renormalization. For $N$ fermions the bounds are proportional to $N$, but for bosons they are sublinear, which implies 'binding', and hence that 'free' bosons are never free. Both 'relativistic' and non-relativistic kinematics are considered. Our bounds are non-perturbative and differ significantly from the predictions of perturbation theory.
\end{abstract}

\section{Introduction}

Quantum electrodynamics (QED), the theory of electrons interacting with photons (at least for small energies) is one of the great successes of physics. Among its major achievements is the explanation of the Lamb shift and the anomalous magnetic moment of the electron. Nevertheless, its computations, which are entirely based on perturbation theory, created some uneasiness among the practitioners. The occurrence of infinities was and is especially vexing. Moreover, a truly nontrivial, 3+1-dimensional example of a relativistically invariant field theory has not yet been achieved.

There are, however, unresolved issues at a much earlier stage of QED that hark back to black-body radiation, the simplest and historically first problem involving the interaction of matter with radiation. The conceptual problems stemming from black-body radiation were partly resolved by quantum mechanics, i.e., by the the non-relativistic Schrödinger equation, which is, undoubtedly, one of the most successful of theories, for it describes matter at low energies almost completely. It is mathematically consistent and there are techniques available to compute relevant quantities. Moreover, it allows us to explain certain facts about bulk matter such as its stability, it extensivity, and the existence of thermodynamic functions. What has not been as successful, so far, is the incorporation of radiation phenomena, the very problem quantum mechanics set out to explain.

It ought to be possible to find a mathematically consistent theory, free of infinities, that describes the interaction of non-relativistic matter with radiation at moderate energies, such as atomic binding energies. It should not be necessary, as

1991 Mathematics Subject Classification. Primary 81V10, 81V70; Secondary 81V45, 81T08, $81 \mathrm{~T} 16$

The first author was supported in part by NSF Grant PHY-95 13072-A02.

The second author was supported in part by NSF Grant DMS-95 00840.

(C) 1999 by the authors. Reproduction of this work, in its entirety, by any means, is permitted for non-commercial purposesby any means is permitted for non-commercial purposes.

(C)0000 (copyright holder) 
some physicists believe, to embed QED as a low energy part of a consistent high energy theory.

From such a theory one could learn a number of things that have not been explained rigorously. i) The decay of excited states in atoms. This problem has been investigated in some ultraviolet cutoff models in [BFS] and in a massive photon model in $[\mathbf{O Y}]$. See also the review of Hogreve $[\mathbf{H}]$. ii) Non-relativistic QED could be a playground for truly non-perturbative calculations and it could shed light on renormalization procedures. In fact, this was the route historically taken by Kramers that led to the renormalization program of Dyson, Feynman, Schwinger and Tomonaga. iii) Last but not least, one could formulate and answer the problems of stability of bulk matter interacting with the radiation field.

It has been proved in $[\mathbf{F}],[\mathbf{L L S}]$ that stability of non-relativistic matter (with the Pauli Hamiltonian) interacting with classical magnetic fields holds provided that the fine-structure constant, $\alpha=e^{2} / \hbar c$, is small enough. It is certain, that the intricacies and difficulties of this classical field model will persist and presumably magnify in QED.

The same may be expected from a relativistic QED since replacing the Pauli Hamiltonian by a Dirac operator leads to a similar requirement on $\alpha$ [LSS]. Indeed, stability of matter in this model (the Brown-Ravenhall model) requires that the electron (positron) be defined in terms of the positive (negative) spectral subspace of the Dirac operator with the magnetic vector potential $A(x)$, instead of the free Dirac operator without $A(x)$. This observation, that perturbation theory, if there is one, must start from the dressed electrons rather than the electrons unclothed by its magnetic field, might ultimately be important in a non-perturbative QED.

The first, humble step is to understand electrons that interact with the radiation field but which are free otherwise. In order for this model to make sense an ultraviolet cutoff has to be imposed that limits the energy of photon modes. The simplest question, which is the one we address in this paper, is the behavior of the self-energy of the electron as the cutoff tends to infinity (with the bare mass of the electron fixed). The self-energy of the electron diverges as the cutoff tends to infinity and it has to be subtracted for each electron in any interacting theory. The total energy will still depend strongly on the cutoff because of the interactions. This dependence will, hopefully, enter through an effective mass which will be set equal to the physical mass (mass renormalization). The resulting theory should be essentially Schrödinger's mechanics, but slightly modified by so-called radiative corrections.

Lest the reader think that the self-energy problem is just a mathematical exercise, consideration of the many-body problem will provide a counterexample. Imagine $N$ charged bosons interacting with the radiation field, but neglect any interaction among them such as the Coulomb repulsion. We say that these particles bind if the energy of the combined particles is less than the energy of infinitely separated particles. As we shall show, charged bosons indeed bind and they do it in such a massive way that it will be very likely that this cannot be overcome by the Coulomb repulsion. In particular, the energy of a charged many-boson system is not extensive, and from this perspective it is fortunate that stable, charged bosons do not exist in nature.

The situation is very different for fermions. We are not able to show that they do not bind but we can show - and this is one of the main results of our paper 
— that the self-energy is extensive, i.e., bounded above and below by a constant times $N$.

We thus have strong evidence that there is no consistent description of a system of stable charged relativistic or non-relativistic bosons interacting with the radiation field, while the Pauli exclusion principle, on the other hand, is able to prevent the above mentioned pathology.

In the remainder of the section we explain our notation and state the results. In the subsequent sections we sketch the proof of some of them but for details we refer the reader to [LL].

We measure the energy in units of $m c^{2}$ where $m$ is the bare mass of the electron, the length in units of the Compton wave length $\ell_{C}=\hbar / m c$ of the bare electron. We further choose $\ell_{C}^{-1} \sqrt{\hbar c}$ as the unit for the vector potential $A$ and $\ell_{C}^{-2} \sqrt{\hbar c}$ as the unit for the magnetic field $B$. The argument is the dimensionless quantity $\ell_{C}^{-1} x$. As usual, $\alpha=e^{2} / \hbar c \approx 1 / 137.04$ is the fine structure constant.

In the expression below, $A(x)$ denotes an ultraviolet cutoff radiation field localised in a box $L \times L \times L$ with volume $V=L^{3}$,

$$
A(x)=\frac{1}{\sqrt{2 V}} \sum_{|k|<\Lambda} \sum_{\lambda=1,2} \frac{1}{\sqrt{|k|}} \varepsilon_{\lambda}(k)\left[a_{\lambda}(k) e^{i x \cdot k}+a_{\lambda}^{*}(k) e^{-i x \cdot k}\right] .
$$

The index $k=2 \pi n / L$ where $n \in \mathbb{Z}^{3}$, and the word cutoff refers to the restriction to all values of $k$ with $|k|<\Lambda$.

The vectors $\varepsilon_{\lambda}(k)$ are the polarization vectors and are normalized in such a way that

$$
\varepsilon_{i}(k) \cdot \varepsilon_{j}(k)=\delta_{i, j}, \varepsilon_{i}(k) \cdot k=0 .
$$

The operators $a_{\lambda}(k)$ and $a_{\lambda}^{*}(k)$ satisfy the commutation relation

$$
\left[a_{\lambda}(k), a_{\lambda^{\prime}}^{*}\left(k^{\prime}\right)\right]=\delta_{\lambda, \lambda^{\prime}} \delta\left(k, k^{\prime}\right),
$$

while all others commute with each other.

The energy of the radiation field can now be conveniently written as

$$
H_{f}=\sum_{|k|<\Lambda} \sum_{\lambda=1,2}|k| a_{\lambda}^{*}(k) a_{\lambda}(k) .
$$

These operators act on the Hilbert space generated by the polynomials in $a_{\lambda}^{*}(k)$ acting on the vacuum $|0\rangle$.

The self energy of (one or more) particles is the ground state energy of the Hamiltonian

$$
H=\text { kinetic energy }+H_{f} .
$$

where, as usual, the ground state energy of $H$ is defined to be

$$
E_{0}=\inf _{\Psi} \frac{\langle\Psi, H \Psi\rangle}{\langle\Psi, \Psi\rangle} .
$$

The first result concerns the self energy of a nonrelativistic electron interacting with the radiation field. The Hamiltonian is given by

$$
H=\frac{1}{2}(p+\sqrt{\alpha} A(x))^{2}+H_{f},
$$

where $p=-i \nabla$ and acts on $L^{2}\left(\mathbb{R}^{3}\right) \otimes \mathcal{F}$, where $\mathcal{F}$ denotes the photon Fock space. 
THEOREM 1.1. The ground state energy, $E_{0}$, of the operator (1.7) satisfies the bounds

$$
C_{1} \alpha^{1 / 2} \Lambda^{3 / 2}<E_{0}<C_{2} \alpha^{2 / 7} \Lambda^{12 / 7}
$$

Let us remark that this result shows that perturbation theory, which predicts $\alpha \Lambda^{2}$ to leading order, is not valid - even for $N=1$.

We do not know how to get upper and lower bounds that are of the same order in $\Lambda$, but we suspect that $\Lambda^{12 / 7}$ is the right exponent. This is supported by the following theorem in which the $p \cdot A$ term is omitted.

THEOREM 1.2. The ground state energy $E_{0}$ of the operator

$$
\frac{1}{2}\left[p^{2}+\alpha A(x)^{2}\right]+H_{f}
$$

satisfies the bounds

$$
C_{1} \alpha^{2 / 7} \Lambda^{12 / 7} \leq E_{0} \leq C_{2} \alpha^{2 / 7} \Lambda^{12 / 7}
$$

While these results are not of direct physical relevance (since $E_{0}$ is not observable), the many-body problem is of importance since it reveals a dramtic difference between bosons and fermions.

THEOREM 1.3. The ground state energy of $N$ bosons, $E_{0}^{\text {boson }}(N)$, with Hamiltonian

$$
H(N)=\sum_{j=1}^{N} \frac{1}{2}\left(p_{j}+\sqrt{\alpha} A\left(x_{j}\right)\right)^{2}+H_{f}
$$

satisfies the bounds

$$
C_{1} \sqrt{N} \sqrt{\alpha} \Lambda^{3 / 2} \leq E_{0}^{\text {boson }}(N) \leq C_{2} N^{5 / 7} \alpha^{2 / 7} \Lambda^{12 / 7}
$$

Thus, the energy $E_{0}^{\text {boson }}(N)$ is not extensive, i.e., it costs a huge energy to separate bosons. This has to be contrasted with the next theorem about fermions. The Hamiltonian is the same as before but it acts on the Hilbert space

$$
\mathcal{F} \otimes \wedge_{j=1}^{N} L^{2}\left(\mathbb{R}^{3} ; \mathbb{C}^{2}\right)
$$

where the wedge product indicates the antisymmetric tensor product is taken.

THEOREM 1.4. The ground state energy, $E_{0}^{\text {fermion }}(N)$, of $N$ charged fermions interacting with the radiation field satisfies

$$
C_{1} \alpha^{1 / 2} \Lambda^{3 / 2} N \leq E_{0}^{\text {fermion }}(N) \leq C_{2} \alpha^{2 / 7} \Lambda^{12 / 7} N
$$

The "relativistic" kinetic energy for an electron is

$$
T^{\mathrm{rel}}=|p+\sqrt{\alpha} A(x)|=\sqrt{[p+\sqrt{\alpha} A(x)]^{2}}
$$

with $p=-i \nabla$. (Really, we should take $\sqrt{[p+\sqrt{\alpha} A(x)]^{2}+1}$, but since $x<$ $\sqrt{x^{2}+1}<x+1$, the difference is bounded by $N$.) 
Consider, first, the $N=1$ body problem with the Hamiltonian

$$
H=T^{\mathrm{rel}}+H_{f} \text {. }
$$

By simple length scaling (with a simultaneous scaling of the volume $V$ ) we easily see that $E_{0}=\inf \operatorname{spec}(H)=C \Lambda$. Our goal here is to show that the constant, $C$, is strictly positive and to give an effective lower bound for it. But we would like to do more, namely investigate the dependence of this constant on $\alpha$. We also want to show, later on, that for $N$ fermions the energy is bounded below by a positive constant times $N \Lambda$. Our proof will contain some novel - even bizarre - features.

TheOREM 1.5. For the Hamiltonian in 1.16 there are positive constants, $C, C^{\prime}, C^{\prime \prime}$ such that

$$
\begin{aligned}
& E_{0} \leq C \sqrt{\alpha} \Lambda \\
& E_{0} \geq C^{\prime} \sqrt{\alpha} \Lambda \text { for small } \alpha \\
& E_{0} \geq C^{\prime \prime} \Lambda \text { for large } \alpha .
\end{aligned}
$$

The generalization of this to $N$ fermions is similar to the nonrelativistic generalization, except that the power of $\Lambda$ is the same on both sides of the inequalities.

Theorem 1.6. For $N$ fermions with Hamiltonian

$$
H_{N}=\sum_{i=1}^{N} T^{\mathrm{rel}}\left(x_{i}\right)+H_{f}
$$

there are positive constants $C, C^{\prime}, C^{\prime \prime}$, independent of $\alpha$ and $N$, such that

$$
\begin{aligned}
& E_{0} \leq C N \sqrt{\alpha} \Lambda \\
& E_{0} \geq C^{\prime} N \sqrt{\alpha} \Lambda \text { for small } \alpha \\
& E_{0} \geq C^{\prime \prime} N \Lambda \text { for large } \alpha
\end{aligned}
$$

We close this introduction by mentioning one last result about the Paulioperator. The kinetic energy expression is given by

$$
T^{\text {Pauli }}=[\sigma \cdot(p+\sqrt{\alpha} A(x))]^{2}=(p+\sqrt{\alpha} A(x))^{2}+\sqrt{\alpha} \sigma \cdot B(x) .
$$

where $\sigma$ denotes the vector consisting of the Pauli matrices. Observe that this term automatically accounts for the spin-field interaction. Our result for the self energy of a Pauli electron is the following.

THEOREM 1.7. The ground state energy $E_{0}$ of the Hamiltonian with Pauli energy,

$$
\frac{1}{2}[\sigma \cdot(p+\sqrt{\alpha} A(x))]^{2}+H_{f}
$$

satisfies the bounds

$$
\begin{aligned}
& E_{0} \leq C_{3} \sqrt{\alpha} \Lambda^{3 / 2} \\
& E_{0} \geq C_{1} \alpha \Lambda \text { for small } \alpha \\
& E_{0} \geq C_{2} \alpha^{1 / 3} \Lambda \text { for large } \alpha
\end{aligned}
$$

For $N$ fermions, the bounds above are multiplied by $N$ (and the constants are changed). 
For the detail of the proof, we refer the reader to [LL]. We believe that the upper bound is closer to the truth since the main contributions to the self energy should come from the fluctuations of the $A^{2}$ term.

Theorem1.7 has the following consequence for stability of matter interacting with quantized fields. It was shown in [LLS] that a system of electrons and nuclei interacting with Coulomb forces, with the Pauli kinetic energy for the electrons and with a classical magnetic field energy is stable (i.e., the ground state energy is bounded below by $N$ ) if and only if $\alpha$ is small enough. In [BFG],[FFG] this result was extended to quantized, ultraviolet cutoff magnetic fields (as here). Among other things, it was shown in $[\mathbf{F F G}]$ that the ground state energy, $E_{0}$, of the electrons and nuclei problem is bounded below by $-\alpha^{2} \Lambda N$ for small $\alpha$. Theorem1.7 implies, as a corollary, that for small $\alpha$ the total energy (including Coulomb energies) is bounded below by $+\alpha \Lambda N$. In other words, among the three components of energy (kinetic, field and Coulomb), the first two overwhelm the third - for small $\alpha$, at least.

All of these statements are true without mass renormalization and the situation could conceivably be more dramatic when the mass is renormalized. In any case, the true physical questions concern energy differences, and this question remains to be addressed.

\section{NON-RELATIVISTIC ENERGY BOUNDS}

Theorem 1.1: We sketch a proof of Theorem1.1. It is clear by taking the state $V^{-1 / 2} / \otimes|0\rangle$ that the ground state energy is bounded above by (const) $\alpha \Lambda^{2}$, which is the same result one gets from perturbation theory. Since the field energy in this state vanishes, such a computation ignores the tradeoff between the kinetic energy of the electron and the field energy. Thus, it is important to quantify this tradeoff. The main idea is to estimate the field energy in terms of selected modes.

Consider the operators (field modes), parametrized by $y \in \mathbb{R}^{3}$,

$$
L(y)=\frac{1}{\sqrt{2 V}} \sum_{|k|<\Lambda, \lambda} \sqrt{|k|} a_{\lambda}(k) \bar{v}_{\lambda}(k) e^{i k \cdot y}
$$

with some arbitrary complex coefficients $v_{\lambda}(k)$. The following lemma is elementary

Lemma 2.1 .

$$
H_{f} \geq \int w(y) L^{*}(y) L(y) \mathrm{d} y
$$

provided that the functions $v_{\lambda}(k)$ and $w$ are chosen such that, as matrices,

$$
|k| \delta_{\lambda^{\prime}, \lambda} \delta\left(k^{\prime}, k\right) \geq \frac{1}{2 V} \bar{v}_{\lambda}(k) \widehat{w}\left(k-k^{\prime}\right) v_{\lambda^{\prime}}\left(k^{\prime}\right),
$$

or equivalently, that

$$
\sum_{|k|<\Lambda, \lambda} \frac{\left|f_{\lambda}(k)\right|^{2}}{\left|v_{\lambda}(k)\right|^{2}} \geq \sum_{|k|,\left|k^{\prime}\right|<\Lambda, \lambda, \lambda^{\prime}} \frac{1}{2 V} \overline{f_{\lambda}(k)} f_{\lambda^{\prime}}\left(k^{\prime}\right) \widehat{w}\left(k-k^{\prime}\right)
$$

for all $f_{\lambda}(k)$, where $\widehat{w}(k)=\int e^{i k \cdot x} w(x) \mathrm{d} x$.

For the proof, one simply notes that both sides of (2.2) are quadratic forms in the creation and annihilation operators, and hence (2.3) and (2.4) are necessary and sufficient conditions for (2.2) to be true. 
Corollary 2.2 .

$$
H_{f} \geq-\frac{1}{2 V} \sum_{|k|<\Lambda, \lambda}|k|\left|v_{\lambda}(k)\right|^{2} \int w(y) \mathrm{d} y+\frac{1}{4}\left\{\begin{array}{l}
\int w(y)\left(L(y)+L^{*}(y)\right)^{2} \mathrm{~d} y \\
-\int w(y)\left(L(y)-\frac{1}{4} L^{*}(y)\right)^{2} \mathrm{~d} y
\end{array}\right.
$$

To prove this, note that

$$
L^{*} L=L L^{*}-\frac{1}{2 V} \sum_{|k|<\Lambda, \lambda}|k|\left|v_{\lambda}(k)\right|^{2},
$$

and, quite generally for operators,

$$
L L+L^{*} L^{*} \leq L^{*} L+L L^{*} .
$$

Returning to the proof of Theorem1.1 we start with the lower bound. Denote by

$$
\Pi(x)=\frac{-i}{\sqrt{2 V}} \sum_{|k|<\Lambda, \lambda} \sqrt{|k|} \varepsilon_{\lambda}(k)\left(a_{\lambda}(k) e^{i k \cdot x}-a_{\lambda}^{*}(k) e^{-i k \cdot x}\right) .
$$

This operator is canonically conjugate to $A(x)$ in the sense that we have the commutation relations

$$
i\left[\Pi_{i}(x), A_{j}(x)\right]=\delta_{i, j} \frac{1}{(3 \pi)^{2}} \Lambda^{3} .
$$

For our calculation, it is important to note the

$$
\operatorname{div} \Pi(x)=0 .
$$

Hence from (2.9) and (2.10) we get that (for all $j$ )

$$
\left[p_{j}+\sqrt{\alpha} A_{j}(x), \Pi_{j}(x)\right]=\sqrt{\alpha} \frac{i}{(3 \pi)^{2}} \Lambda^{3} .
$$

The inequality

$$
\frac{1}{2}(p+\sqrt{\alpha} A(x))^{2}+2 a^{2} \Pi(x)^{2} \geq-a i \sum_{j=1}^{3}\left[p_{j}+\sqrt{\alpha} A_{j}(x), \Pi_{j}(x)\right],
$$

valid for all positive numbers $a$, yields

$$
\frac{1}{2}(p+\sqrt{\alpha} A(x))^{2}+H_{f} \geq a \sqrt{\alpha} \frac{1}{(3 \pi)^{2}} \Lambda^{3}+H_{f}-2 a^{2} \Pi(x)^{2} .
$$

Now, with

$$
v_{\lambda}(k)=(3 \pi) \frac{\varepsilon_{\lambda}(k)}{\Lambda^{3 / 2}}
$$

and

$$
w(y)=\delta(x-y),
$$

it is elementary to see that (2.4) is satisfied. Hence Corollary 2.2 yields

$$
H_{f} \geq \frac{9 \pi^{2}}{4 \Lambda^{3}} \Pi(x)^{2}-\frac{9}{8} \Lambda
$$


Choosing $a=(3 \pi) /\left(\sqrt{2} \Lambda^{3 / 2}\right)$ yields the lower bound

$$
H \geq \frac{1}{3 \pi} \sqrt{\frac{\alpha}{2}} \Lambda^{3 / 2}-\frac{9}{8} \Lambda
$$

The idea of using a commutator, as in (2.12), (2.13) to estimate the ground state energy, goes back to the study of the polaron $[\mathbf{L Y}]$.

For the upper bound we take a simple trial function of the form

$$
\phi(x) \otimes \Psi
$$

where $\Psi \in \mathcal{F}$ is normalized and $\phi(x)$ is a real function normalized in $L^{2}\left(\mathbb{R}^{3}\right)$. An upper bound to the energy is thus given by

$$
\frac{1}{2} \int|\nabla \phi(x)|^{2} \mathrm{~d} x+\frac{\alpha}{2} \int \phi(x)^{2}\left(\Psi, A(x)^{2} \Psi\right) \mathrm{d} x+\left(\Psi, H_{f} \Psi\right) .
$$

It is not very difficult to see that the last two terms can be concatenated into the following expression.

$$
\frac{1}{2} \int\left(\Psi,\left[\Pi(x)^{2}+\alpha A(x)\left(-\Delta+\phi(x)^{2}\right) A(x)\right] \Psi\right) \mathrm{d} x-\frac{1}{2} \operatorname{Tr} \sqrt{P-\Delta P} .
$$

Here, $P$ is the projection onto the divergence free vector fields with ultraviolet cutoff $\Lambda$. This can be deduced by writing the field energy in terms of $\Pi(x)$ and $A(x)$. The first term in (2.20) is a sum of harmonic oscillators whose zero point energy is given by

$$
\frac{1}{2} \operatorname{Tr} \sqrt{P\left(-\Delta+\alpha \phi(x)^{2}\right) P}
$$

and hence

$$
\frac{1}{2} \operatorname{Tr} \sqrt{P\left(-\Delta+\alpha \phi(x)^{2}\right) P}-\frac{1}{2} \operatorname{Tr} \sqrt{P(-\Delta) P},
$$

is an exact expression for the ground state energy. Using the operator monotonicity of the square root we get as an upper bound on (2.20)

$$
\frac{1}{2} \int|\nabla \phi(x)|^{2} \mathrm{~d} x+\frac{1}{2} \sqrt{\alpha} \operatorname{Tr} \sqrt{P \phi(x)^{2} P} .
$$

As a trial function we use the positive function

$$
\phi(x)=\text { const. } K^{-3 / 2} \int\left(1-\frac{|k|}{K}\right)_{+}^{3} e^{i k \cdot x} \mathrm{~d} x .
$$

Optimizing the resulting expression over $K$ yields the stated result. For details we refer the reader to $[\mathbf{L L}]$.

It is natural to ask, how good this upper bound is. If we neglect the cross terms in $(p+A)^{2}$, i.e., we replace the kinetic energy by $p^{2}+\alpha A(x)^{2}$, then we have Theorem 1.2, which we prove next.

Theorem 1.2: The upper bound was already given in Theorem 1.1 because $\langle p \cdot A\rangle=0$ in the state (2.18). Loosely speaking equation (2.9) expresses the Heisenberg uncertainty principle for the field operators. An uncertainty principle that is quite a bit more useful is the following. 
LEMMA 2.3. The following inequality holds in the sense of quadratic forms

$$
\Pi(x)^{2} \geq \frac{1}{4} \frac{1}{(3 \pi)^{4}} \Lambda^{6} \frac{1}{A(x)^{2}} \text {. }
$$

For the proof note that $\left[A_{j}(x), A_{k}(y)\right]=0$ and compute

$$
i\left[\Pi(x)_{j}, \frac{A_{j}(x)}{A(x)^{2}}\right]=\frac{1}{(3 \pi)^{2}} \Lambda^{3}\left[\frac{1}{A(x)^{2}}-2\left(\frac{A_{j}(x)}{A(x)^{2}}\right)^{2}\right],
$$

and summing over $j$ we obtain that

$$
i \sum_{j=1}^{3}\left[\Pi(x)_{j}, \frac{A_{j}(x)}{A(x)^{2}}\right]=\frac{1}{(3 \pi)^{2}} \Lambda^{3} \frac{1}{A(x)^{2}}
$$

Our statement follows from the Schwarz inequality.

To prove Theorem 1.2 we return to Lemma 2.1 and choose $v_{\lambda}(k)=\varepsilon_{\lambda}(k)$ and $w(x)$ any function $\leq 1$. Corollary 2.2 applied to each of the 3 components of $\Pi(x)$ then yields

$$
H_{f} \geq \frac{1}{4} \int w(x-y) \Pi(y)^{2} \mathrm{~d} y-\Lambda^{4} \frac{3}{8 \pi^{2}} \int w(y) \mathrm{d} y,
$$

for every $x \in \mathbb{R}^{3}$. By Lemma 2.1 the right side is bounded below by

$$
\Lambda^{6} \int w(x-y) \frac{1}{A(y)^{2}} \mathrm{~d} y-\Lambda^{4} \int w(y) \mathrm{d} y,
$$

and hence

$$
\begin{aligned}
\langle\Psi, H \Psi\rangle & \geq \frac{1}{2} \int\langle\nabla \Psi(x), \nabla \Psi(x)\rangle \mathrm{d} x+\frac{\alpha}{2} \int\left\langle\Psi(x), A(x)^{2} \Psi(x)\right\rangle \mathrm{d} x \\
& +\Lambda^{6} \int w(x-y)\left\langle\Psi(y), \frac{1}{A(x)^{2}} \Psi(y)\right\rangle \mathrm{d} y \mathrm{~d} x \\
& -\Lambda^{4} \int w(y) \mathrm{d} y \int\langle\Psi(x), \Psi(x)\rangle \mathrm{d} x .
\end{aligned}
$$

By Schwarz's inequality the second and third term together are bounded below by

$$
\sqrt{\frac{\alpha}{2}} \Lambda^{3} \int\langle\Psi(x), \Psi(y)\rangle \frac{w(x-y)}{\sqrt{\int w(z) \mathrm{d} z}} \mathrm{~d} x \mathrm{~d} y .
$$

If we restate our bound in terms of Fourier space variables we get

$$
\int\left[\frac{|p|^{2}}{2}+\sqrt{\frac{\alpha}{2}} \Lambda^{3} \frac{\widehat{w}(p)}{\sqrt{\widehat{w}(0)}}\right]\langle\widehat{\Psi}(p), \widehat{\Psi}(p)\rangle \mathrm{d} p-\Lambda^{4} \widehat{w}(0) \int\langle\widehat{\Psi}(p), \widehat{\Psi}(p)\rangle \mathrm{d} p .
$$

Choosing the function $\widehat{w}(p)$ to be $(2 \pi)^{3} \Lambda^{-18 / 7}$ times the characteristic function of the ball of radius $\Lambda^{6 / 7}$, we have that $w(x) \leq 1$ and it remains to optimize $(2.32)$ over all normalized states $\widehat{\Psi}(p)$. This is easily achieved by noting that the function

$$
\frac{1}{2}|p|^{2}+\sqrt{\frac{\alpha}{2}} \Lambda^{3} \frac{\widehat{w}(p)}{\sqrt{\widehat{w}(0)}}
$$

is everywhere larger than $\Lambda^{12 / 7}$. Strictly speaking, the function $w(x)$ should be positive in order for the argument that led to $(2.31)$ to be valid. This can be 
achieved with a different choice of $w(x)$ that is more complicated but does not change the argument in an essential way.

\section{NON-RELATIVISTIC MANY-BODY ENERGIES}

A problem that has to be addressed is the energy of $N$ particles (bosons or fermions) interacting with the radiation field. If $E_{0}=E_{0}(1)$ is the energy of one particle (which we estimated in the preceding section) then, ideally the energy, $E_{0}(N)$, of $N$ particles (which trivially satisfies $E_{N} \leq N E$, since the $N$ particles can be placed infinitely far apart) ought to be, exactly,

$$
E_{0}(N)=N E_{0}
$$

in a correct QED. In other words, in the absence of nuclei and Coulomb potentials, there should be no binding caused by the field energy $H_{f}$. This is what we seem to observe experimentally, but this important topic does not seem to have been discussed in the QED literature.

Normally, one should expect binding, for the following mathematical reason: The first particle generates a field, $A(x)$, and energy $E_{0}$. The second particle can either try to generate a field $A(x+y)$, located very far away at $y$ or the second particle can try to take advantage of the field $A(x)$, already generated by the first particle, and achieve an insertion energy lower than $E_{0}$.

Indeed, this second phenomenon happens for bosons - as expected. For fermions, however, the Paul principle plays a crucial role (even in in the absence of Coulomb attractions). We show that $E_{0}(N) \geq C N E_{0}$ for fermions, but we are unable to show that the universal constant $C=1$. Even if $C<1$, the situation could still be saved by mass renormalization, which drives the bare mass to zero as $\Lambda$ increases, thereby pushing particles apart.

3.1. BOSONS. Theorem 1.3; This theorem concerns the ground state energy of $N$ charged bosons. the Hamiltonian is given by 1.11 acting on the Hilbert space of symmetric functions tensored with the photon Fock space $\mathcal{F}$. It states, basically, that $C_{1} \sqrt{N} \sqrt{\alpha} \Lambda^{3 / 2} \leq E_{0}^{\text {boson }}(N) \leq C_{2} N^{5 / 7} \alpha^{2 / 7} \Lambda^{12 / 7}$.

The proof follows essentially that of the one particle case. The interesting fact is that it implies binding of charged bosons (in the absence of the Coulomb repulsion). The binding energy is defined by

$$
\Delta E(N)=E_{0}(N)-N E_{0}(1)
$$

and satisfies the bounds

$$
\begin{aligned}
& \Delta E(N) \geq C_{1} \sqrt{N} \sqrt{\alpha} \Lambda^{3 / 2}-C_{2} N \alpha^{2 / 7} \Lambda^{12 / 7} \\
& \Delta E(N) \leq C_{2} N^{2 / 7} \alpha^{2 / 7} \Lambda^{12 / 7}-C_{1} N \sqrt{\alpha} \Lambda^{3 / 2}
\end{aligned}
$$

which can be made negative for appropriately chosen $N$ and $\Lambda$. There will be binding for all large enough $N$, irrespective of the cutoff $\Lambda$. It also has to be remarked that the Coulomb repulsion will, in all likelihood, not alter this result since it has an effect on energy scales of the order of $\Lambda$ and not $\Lambda^{12 / 7}$ or $\Lambda^{3 / 2}$.

3.2. FERMIONS. The real issue for physics is what happens with fermions. We cannot show that there is no binding but we can show that the energy is extensive as in Theorem 1.4. The Hamiltonian is the same as (1.11) but it acts on antisymmetric functions tensored with $\mathcal{F}$. (Spin can be ignored for present purposes.) 
Rough sketch of the proof of Theorem 1.11.

The difficulty in proving this theorem stems from the fact that the field energy is not extensive in any obvious way.

Define $\underline{\mathrm{X}}=\left(x_{1}, \cdots, x_{N}\right)$ and define the function

$$
n_{j}(\underline{\mathrm{X}}, R)=\#\left\{x_{i} \neq x_{j}:\left|x_{i}-x_{j}\right|<R\right\} .
$$

This function counts the number of electrons that are within a distance $R$ of the $j^{\text {th }}$ electron. Note that this function is not smooth, so that all the following computations have to be modified. (See $[\mathbf{L L}]$.) We save half of the kinetic energy and write

$$
H=\frac{1}{4} \sum_{j=1}^{N}\left(p_{j}+\sqrt{\alpha} A\left(x_{j}\right)\right)^{2}+H^{\prime} .
$$

We apply the commutator estimate (2.11) to the pair

$$
i\left[p_{j}+\sqrt{\alpha} A\left(x_{j}\right), \frac{1}{\sqrt{N_{j}(\underline{X}, R)+1}} \Pi\left(x_{j}\right)\right]
$$

and obtain the bound (with the caveat mentioned above), for all $\alpha>0$,

$$
H^{\prime} \geq a \sqrt{\alpha} \Lambda^{3} \sum_{j=1}^{N} \frac{1}{\sqrt{N_{j}(\underline{\mathrm{X}}, R)+1}}-a^{2} \sum_{j=1}^{N} \frac{1}{N_{j}(\underline{\mathrm{X}}, R)+1} \mathrm{~F}\left(x_{j}\right)^{2}+H_{f}
$$

The next two steps are somewhat nontrivial and we refer the reader to [LL]. First one notes that the modes $F\left(x_{i}\right)$ and $F\left(x_{j}\right)$ are essentially orthogonal (i.e., they commute) if $\left|x_{i}-x_{j}\right|>\Lambda^{-1}$. Ignoring the technical details of how this is implemented, the key observation is that the last two terms in (3.3) can be estimated from below by $-N \Lambda$ provided $a=\Lambda^{-3 / 2}$.

The next ingredient is a new Lieb-Thirring type estimate involving the function $N_{j}(\underline{\mathrm{X}}, R)$. It is here and only here that the Pauli exclusion principle is invoked.

Theorem 3.1. On the space $\left.\wedge_{j=1}^{N} L^{2} \mathbb{R}^{3} ; \mathbb{C}^{q}\right)$ of antisymmetric functions

$$
\sum_{j=1}^{N}\left(p_{j}+\sqrt{\alpha} A\left(x_{j}\right)\right)^{2} \geq \frac{C}{q^{2 / 3}} \frac{1}{R^{2}} \sum_{j=1}^{N} N_{j}(\underline{X}, R)^{2 / 3}
$$

with $C \geq 0.00127$. An analogous inequality holds for the relativistic case as well:

$$
\sum_{j=1}^{N}\left|p_{j}+\sqrt{\alpha} A\left(x_{j}\right)\right| \geq \frac{C}{q^{1 / 3}} \frac{1}{R} \sum_{j=1}^{N} N_{j}(\underline{X}, R)^{1 / 3}
$$

By using the kinetic energy previously saved together with (3.3) and the previous discussion, we get

$$
H \geq \sum_{j=1}^{N}\left\{N_{j}(\underline{\mathrm{X}}, R)^{2 / 3}+\sqrt{\alpha} \Lambda^{3 / 2} \frac{1}{\sqrt{N_{j}(\underline{\mathrm{X}}, R)+1}}\right\}-N \Lambda .
$$

By minimizing over $N_{j}$ the desired estimate is obtained. The upper bound is fairly elementary and is omitted. 


\section{RELATIVISTIC ENERGY BOUNDS}

Theorem 1.5: Sketch of Proof.

An upper bound for $E_{0}$ is easy to obtain, but it is indirect. Note that

$$
|p+\sqrt{\alpha} A(x)| \leq \varepsilon[p+\sqrt{\alpha} A(x)]^{2}+(4 \varepsilon)^{-1}
$$

for any $\varepsilon>0$. Take $\Psi=f(x) \otimes|0\rangle$ with $|0\rangle$ being the Fock space vacuum. Using $(4.1)$

$$
\begin{aligned}
(\Psi, H \Psi) & \leq \varepsilon \int_{\mathbb{R}^{3}}\left\{\alpha\left\langle 0\left|A(x)^{2}\right| 0\right\rangle|f(x)|^{2}+|\nabla f(x)|^{2}\right\} d x+\varepsilon^{-1} \\
& =\frac{\varepsilon \alpha \Lambda^{2}}{4 \pi}+\int|\nabla f|^{2}+\frac{1}{4 \varepsilon},
\end{aligned}
$$

since $\left\langle 0\left|A(x)^{2}\right| 0\right\rangle=(2 \pi)^{-3} \int_{|k|<\Lambda}|k|^{-1} d k=\Lambda^{2} / 4 \pi^{2}$. We can now let $f(x) \rightarrow V^{-\frac{1}{2}}$ and take $\varepsilon=(\pi / \alpha)^{1 / 2} \Lambda^{-1}$, whence

$$
E_{0} \leq(\alpha / 4 \pi)^{1 / 2} \Lambda
$$

Now we turn to the lower bound for $H$.

Step 1: Since $x \rightarrow \sqrt{x}$ is operator monotone,

$$
T>T_{1}=\left|p_{1}+\sqrt{\alpha} A_{1}(x)\right|,
$$

where the subscript 1 denotes the 1 component (i.e., the $x$-component) of a vector. By replacing $T$ by $T_{1}$, we are now in a position to remove $A_{1}$ by a gauge transformation - but it has to be an operator-valued gauge transformation. The use of such a gauge transformation is is a novelty, as far as we are aware, in QED.

To effect the gauge transformation, set

$$
\varphi(x)=\frac{1}{\sqrt{2 V}} \sum_{h, \lambda} \frac{\varepsilon^{\lambda}(k)_{1}}{\sqrt{(k)}}\left[a_{\lambda}(k)+a_{\lambda}^{*}(-k)\right] \frac{e^{i k_{1} x_{1}}-1}{i k_{1}} e^{i k_{\perp} x_{\perp}}
$$

with $x_{\perp}=\left(x_{2}, x_{3}\right)$. Then $\left[A_{j}(x), \varphi(x)\right]=0, j=1,2,3$ and $p_{1} \exp [i \varphi(x)]=$ $-A_{1}(x)$. The unitary $U=\exp [i \varphi(x)]$ is a gauge transformation, but it is operatorvalued. We have

$$
\begin{aligned}
U^{-1}\left|p_{1}+A_{1}(x)\right| U & =\left|p_{1}\right| \\
U^{-1} a_{\lambda}(k) U & =a_{\lambda}(k)+f_{\lambda}(k, x) \\
U^{-1} a_{\lambda}^{*}(k) U & =a_{\lambda}^{*}(k)+\bar{f}_{\lambda}(k, x) \\
\tilde{H}_{f}=U^{-1} H_{f} U & =\sum_{k, \lambda}|k|\left[a_{\lambda}^{*}(k)+\bar{f}_{\lambda}(k, x)\right]\left[a_{\lambda}(k)+f_{\lambda}(k, x)\right]
\end{aligned}
$$

with

$$
f_{\lambda}(k, x)=\sqrt{\frac{\alpha}{2 V}} \sum_{k, \lambda} \frac{\varepsilon_{\lambda}(k)_{1}}{|k|} \frac{e^{-i k_{1} x_{1}}-1}{k_{1}} e^{-i k_{\perp} x_{\perp}} .
$$

Since $p_{\perp}$ does not appear in our new Hamiltonian,

$$
\widetilde{H}=U^{-1} H U=\left|p_{1}\right|+\tilde{H}_{f},
$$

the variable $x_{\perp}$ appears only as a parameter, and thus we can set $x_{\perp}=$ constant $=(0,0)$, by translation invariance, and replace $\mathbb{R}^{3}$ by $\mathbb{R}^{1}=\mathbb{R}$.

From now on $x_{1}=x$ and, $p_{1}=p=-i d / d x$. 
Step 2: The dependence on $x$ now appears in $\tilde{H}_{f}$ instead of in the kinetic energy, $|p|$. For each $x$ we can try to put $\widetilde{H}_{f}$ into its ground state, which is that of a displaced harmonic oscillator. But, since this state depends on $x$, to do so will require a great deal of kinetic energy, $\left|p_{1}\right|$.

Let $\Psi$ be a normalized wave-function, i.e., a function on $L^{2}(\mathbb{R}) \otimes \mathcal{F}$. We write it as $\psi_{x}$ where $\psi_{x} \in \mathcal{F}$. Thus, with $\langle\cdot, \cdot\rangle$ denoting the inner product on $\mathcal{F}, \int_{\mathbb{R}}\left\langle\psi_{x}, \psi_{x}\right\rangle d x=1$.

Decompose $\mathbb{R}$ as the disjoint union of intervals of length $\ell / \Lambda$, where $\ell$ is a parameter to be determined later. Denote these intervals by $I_{j}, j=1,2, \ldots$. A simple Poincaré type inequality gives, for $g: L^{2}(\mathbb{R}) \rightarrow \mathbb{C}$,

$$
(g,|p| g) \geq C_{1} \frac{\Lambda}{\ell} \sum_{j} \int_{I_{j}}\left\{|g(x)|^{2}-\left|\bar{g}_{j}\right|^{2}\right\} d x,
$$

where $\bar{g}_{j}=\frac{\Lambda}{\ell} \int_{I_{j}} g(x) d x$ is the average of $g$ in $I_{j}$. Then

$$
(\Psi,|p| \Psi) \geq C_{1} \frac{\Lambda}{\ell} \sum_{j} \int_{I_{j}}\left\{\left\langle\psi_{x}, \psi_{x}\right\rangle-\left\langle\bar{\psi}_{j}, \bar{\psi}_{j}\right\rangle\right\} d x .
$$

Step 3: Next, we analyze $\tilde{H}_{f}$. We think of this as an operator on $\mathcal{F}$, parameterized by $x \in \mathbb{R}$. We would like $\widetilde{H}_{f}$ to have a gap so we define

$$
H_{x}=\frac{\Lambda}{2} \sum_{\varepsilon \Lambda \leq|k| \leq \Lambda} \sum_{\lambda}\left[a_{\lambda}^{+}(k)+\bar{f}_{\lambda}(k, x)\right] \cdot[\text { h.c. }]
$$

Clearly, $\widetilde{H}_{f} \geq H_{x}$ and

$$
(\Psi, \tilde{H} \Psi) \geq \frac{\Lambda}{\ell} \sum_{j} \int_{I_{j}}\left\langle\psi_{x}, \psi_{x}\right\rangle-\left\langle\bar{\psi}_{j}, \bar{\psi}_{j}\right\rangle+\left\langle\psi_{x}, H_{x} \psi_{x}\right\rangle d x .
$$

For each interval $I_{j}$ we can minimize (4.11) subject to $\int_{I_{j}}\left\langle\psi_{x}, \psi_{x}\right\rangle d x$ fixed. This leads to

$$
\left(h_{j} \psi\right)_{x}=e \psi_{x}
$$

with

$$
\left(h_{j} \psi\right)_{x}=\frac{\Lambda}{\ell} \psi_{x}-\frac{\Lambda}{\ell} \bar{\psi}_{j}+H_{x} \psi_{x}
$$

Obviously, this eigenvalue problem $(4.12,4.13)$ is the same for all intervals $I_{j}$, so we shall drop the subscript $j$ and try to find the minimum $e$.

A lower bound to $h_{j}$ (and hence to $e$ ) can be found by replacing $H_{x}$ by

$$
\widehat{H}_{x}=\frac{\Lambda}{2}\left(1-\Pi_{x}\right),
$$

where $\Pi_{x}=\left|g_{x}\right\rangle\left\langle g_{x}\right|$ is the projector onto the ground state, $\left|g_{x}\right\rangle$, of $H_{x}$.

If we substitute $\widehat{H}_{x}$ into (4.13) the corresponding eigenvalue equation (4.12) becomes soluble. Multiply (4.12) on the left by $\left\langle g_{x}\right|$, whence

$$
\left(\frac{\Lambda}{\ell}-e\right)\left\langle g_{x}, \psi_{x}\right\rangle=\frac{\Lambda}{\ell}\left\langle g_{x}, \bar{\psi}\right\rangle
$$

Then, substitute (4.14) into (4.13) and integrate $\int_{I} d x$ to find 


$$
\frac{1}{2} \Lambda^{3} \ell^{-2}\left(\int \Pi_{x} d x\right) \bar{\psi}=\left(\frac{\Lambda}{\ell}-e\right)\left(\frac{\Lambda}{2}-e\right) \bar{\psi} .
$$

We know that $e<\Lambda / 2$ because we could take $\psi_{x}=$ constant as a trial function, and then use $\tilde{H}_{x} \leq \Lambda / 2$. Also, $e<\Lambda / \ell$, because we could take $\Psi=\delta_{x_{0}}\left|g_{x_{0}}\right\rangle$.

Step 4: Eq. (4.15) will give us a lower bound to $e$ if we can find an upper bound to $Y=(\Lambda / \ell) \int_{I} \Pi_{x} d x$. To do this note that

$$
\begin{aligned}
Y^{2} & \leq \operatorname{Trace} Y^{2}=\left(\frac{\Lambda}{\ell}\right)^{2} \int_{I} \int_{I}\left|\left\langle g_{x}, g_{y}\right\rangle\right|^{2} d x d y \\
& =\left(\frac{\Lambda}{\ell}\right)^{2} \int_{I} \int_{I} \exp \left\{-\frac{\alpha}{2 V} \sum_{\varepsilon \Lambda \leq|k| \leq \Lambda} \sum_{\lambda}\left|f_{\lambda}(k, x)-f_{\lambda}(k, y)\right|^{2} d x d y\right\}
\end{aligned}
$$

Noting that $\sum_{\lambda=1}^{2} e_{\lambda}(k)_{1}^{2}=1-k_{1}^{2} / k^{2}$, the quantity $\{\quad\}$ in (4.16) becomes (as $V \rightarrow \infty)$

$$
\{\quad\}=-\frac{2 \alpha}{(2 \pi)^{3}} \int_{\Lambda / 2<|k|<\Lambda} \frac{1}{|k|^{3} k_{1}^{2}}\left(k_{\perp}^{2}\right)\left[\sin \frac{k_{1}}{2}(x-y)\right]
$$

After some algebra we find that

$$
\left(\frac{1}{\ell}-\frac{e}{\Lambda}\right)\left(\frac{1}{2}-\frac{e}{\Lambda}\right) \leq \frac{1}{2 \ell}\left(\text { Trace } Y^{2}\right)^{1 / 2} \leq \frac{1}{2 \ell} \sqrt{K_{\ell}(\alpha)}
$$

where

$$
\begin{aligned}
K_{\ell}(\alpha) & =\int_{0}^{1} \int_{0}^{1} \exp \left[-\alpha \frac{\ell}{\pi^{2}}|x-y| \int_{0}^{|x-y| \ell / 4}\left(\frac{\sin t}{t}\right)^{2} d t\right] d x d y . \\
& \leq \int_{-1 / 2}^{1 / 2} \exp \left[-\alpha x^{2} \ell^{2} / 8 \pi\right] d x
\end{aligned}
$$

We find that

$$
\begin{aligned}
K_{\ell}(\alpha) & \sim 1-\alpha \ell^{2} / 96 \pi, \quad \ell^{2} \alpha \text { small } \\
& \sim \sqrt{2} \pi\left(\alpha \ell^{2}\right)^{1 / 2}, \quad \ell^{2} \alpha \text { large }
\end{aligned}
$$

If $\alpha$ is small we take $\ell \sim \alpha^{-1 / 2}$. If $\alpha$ is large we take $\ell=2$. This establishes our theorem for $N=1$.

Theorem 1.6: Sketch of Proof.

For $N>1$ we can decompose $\mathbb{R}^{3}$ into cubic boxes $B_{j}, j=1,2,3, \ldots$ of size $\ell \Lambda$ and "borrow" $\frac{1}{2}|p+A(x)|^{2}$ kinetic energy from each particle. That is, $H_{N}=$ $H_{N}^{1 / 2}+\frac{1}{2} T_{N}$ with $T_{N}=\sum_{i=1}^{N} T\left(x_{i}\right)$. The Pauli principle will then yield an energy for $\frac{1}{2} T_{N}$ that is bounded below by (const.) $\left(n_{j-1}\right)^{4 / 3}$, where $n_{j}$ is the particle number in box $B_{j}$.

\section{References}

[BFG] L. Bugliaro, J. Fröhlich and G.M. Graf, Stability of quantum electrodynamics with nonrelativistic matter, Phys.Rev. Lett. 77 (1996), 3494-3497. 
[BFS] V. Bach, J. Fröhlich and I.M. Sigal, Renormalization group analysis of spectral problems in quantum field theory, Adv. Math. 137 (1998),205-298; Quantum electrodynamics of confined nonrelativistic particles, ibid 299-395.

[F] C. Fefferman, Stability of Coulomb systems in a magnetic field, Proc. Natl. Acad. Sci. USA, 92 (1995), 5006-5007.

[FFG] C. Fefferman, J. Fröhlich and G.M. Graf, Stabilty of nonrelativistic quantum mechanical matter coupled to the (ultraviolet cutoff) radiation field, Proc. Natl. Acad. Sci. USA 93 (1996), 15009-15011; Stability of ultraviolet cutoff quantum electrodynamics with non-relativistic matter, Commun. Math. Phys. 190 (1997), 309-330.

[H] H. Hogreve, Math. Reviews 99e:81051a, b Amer. Math. Soc. (1999).

[LL] E.H. Lieb and M. Loss, Remarks about the ultraviolet problem in QED, (in preparation).

[LLS] E.H. Lieb, M. Loss and J. P. Solovej, Stability of Matter in Magnetic Fields, Phys. Rev. Lett. 75, 985-989 (1995).

[LSS] E.H. Lieb, H. Siedentop and J.P. Solovej, Stability and Instability of Relativistic Electrons in Magnetic Fields, J. Stat. Phys. 89 (1997), 37-59.

[LY] E.H.Lieb and K. Yamazaki, Ground State Energy and Effective Mass of the Polaron, Phys. Rev. 111 (1958), 728-733.

[OY] T. Okamoto and K. Yajima, Complex scaling technique in nonrelativistic massive QED, Ann. Inst. H. Poincaré Phys. Théor. 42 (1985), 311-327.

Departments of Mathematics and Physics, Princeton University, Princeton, New JERSEY 08544-0708

E-mail address: lieb@math.princet on.edu

School of Mathematics, Georgia Institute of Technology, Atlanta, Georgia 30332 0160

E-mail address: loss@math.gatech.edu 